\title{
Coagulopathies in Pregnancy: What an Obstetrician Ought to Know!
}

\author{
Abhay A. Bhave ${ }^{1,2,3}$ \\ Received: 8 November 2019 / Accepted: 12 November 2019 / Published online: 27 November 2019 \\ (c) Federation of Obstetric \& Gynecological Societies of India 2019
}

It is a matter of concern if you read " 33 women die every hour from pregnancy and childbirth complications - India among 10 nations with highest maternal mortality ratio" (WHO). Complications of pregnancy and childbirth are the leading causes of disability and death amongst women between the ages of 15 and 49 . In a study conducted in Mumbai, more than half of the women suffer from various complications and were in the younger age hailing from rural areas [1].

In 1990, the WHO reported the death of 523,000 mothers due to complications in pregnancy and childbirth; in 2013, this number was $45 \%$ less indicating that advances in science have helped us achieve this drop in mortality. But we are still falling short as the distribution of health-care talent and mechanisms is discordant between the privileged and the underprivileged.

Haematologic emergencies contribute to significant morbidity and mortality in the pregnant population and can be sudden, unprecedented and catastrophic. Certainly, there is an opportunity to prevent haematological emergencies in our community and we owe it to our women, to do our best as thinkers, planners and executors of good health care and safe practices. So are we delivering the same? I feel that awareness of pathophysiology and anticipation or recognition of possible hazardous events are two keys to better therapy and outcome.

Dr Abhay A Bhave, Consultant haematologist, Lilavati hospital and Research Centre, Bandra West, Mumbai, Global hospitals, Parel, Mumbai, Empire Haematology Oncology Specialty centre, Bandra west, Mumbai.

Abhay A. Bhave

bhaveabhay@hotmail.com

1 Lilavati Hospital and Research Centre, Bandra West, Mumbai, India

2 Global Hospitals, Parel, Mumbai, India

3 Empire Haematology Oncology Specialty Centre, Bandra West, Mumbai, India
Coagulopathies in pregnancy can present or lead to obstetric emergencies and can vary from prothrombotic / microangiopathic events such as HELLP (Haemolysis Elevated Liver Enzymes Low Platelets), TTP (thrombotic thrombocytopenia purpura) and other microangiopathies, especially DIC (disseminated Intravascular coagulopathy), to deep venous thrombosis (DVT) and recurrent pregnancy losses and, on the other hand, bleeding events such as antepartum haemorrhage and PPH (postpartum haemorrhage) just to name a few.

These could be prevented if we monitor the patient well for warning signs that could warrant tests to diagnose these unwanted events. Unfortunately, a regular ANC follow-up in our clinics is not common and sometimes patients present to us for the first time with an emergency with no documentation of previous adverse events in the earlier pregnancy.

So, how can we recognise and prevent such catastrophic events in our patients? Surely, by understanding the pathophysiology we could attempt in all honesty to do so. A good history of past episodes of bleeding along with a family history of bleeding can warn of a bleeding tendency such as a congenital bleeding disorder like von Willebrand disease (prolonged bleeding time, raised APTT (activated partial thromboplastin time) and history of mucosal bleeding from childhood, especially menorrhagia). Simple laboratory tests such as a global coagulation panel including a CBC, PT (prothrombin time), PTT/PTTK (partial thromboplastin time with kaolin) and plasma fibrinogen could predict a coagulopathy. A prolonged PT could suggest underlying liver disease or FVII deficiency, while a prolonged APTT/PTTK could suggest deficiency of the intrinsic pathway (Factor XII, XI, IX, VIII) and a prolongation of both could suggest a defect in the global coagulation system, especially DIC or coagulopathy of PPH or of massive transfusion. A short (less than the control) value of the coagulation tests (PT, APTT/ PTTK) may actually warn us of a prothrombotic state such as preeclampsia or early DIC (disseminated intravascular coagulopathy) in a given clinical situation. TEG (thomboelastogram) is a simple bedside test with a fast turn-around time that may identify and guide appropriate component 
therapy in case of bleeding, especially in a patient with PPH or postoperative state where you would like to differentiate between a coagulopathy versus a local surgical cause for bleeding [2].

Of all the coagulopathies, disseminated intravascular coagulopathy (DIC) is by far the worst as its morbidity and mortality are high. DIC is associated with a systemic activation of coagulation causing disseminated fibrin deposition in the small blood vessels due to activation of thrombin, culminating in multiple organ failure. This results in activation of coagulation resulting in depletion of platelets and coagulation factors, which may cause bleeding (consumption coagulopathy) [3]. DIC can arise from a series of pregnancy complications ranging from acute peripartum haemorrhage to placental abruption to preeclampsia/eclampsia/HELLP to retained stillbirth/ septic and acute fatty liver of pregnancy. Diagnosis of DIC can be made early by awareness of the clinical picture of persistent oozing at surgical site, ecchymosis on the skin, a multisystem organ involvement or failure, changes in the colour of the digits, reducing platelets count and a prolonged PT/ APTT/ PTTK, especially with a dropping fibrinogen (even if it is in the normal range a dropping trend of fibrinogen is significant hence the need for a baseline value) with rising D Dimer or FDP (fibrin degradation products) and schistocytes on smear [4]. Treatment of DIC involves correcting the underlying causes like, controlling: sepsis, removal of the dead foetus, control of bleeding and avoiding unnecessary transfusion, as it can cause a dilution DIC. However, if there is bleeding then use of different blood components or factor therapy PCC (prothrombin complex concentrates) to replete the factors and contain the DIC is the best option to normalise the coagulation profile. The typical target for blood components in a bleeding patient with DIC is: platelet $\geq 0000 \mathrm{~mm}^{3}$, PT within $3 \mathrm{~s}$ of control and APTT/ PTTK within $6 \mathrm{~s}$ of control, haemoglobin at $8 \mathrm{gm} \%$ and fibrinogen more than $1.5 \mathrm{~g} / \mathrm{l}$. DIC can cause significant morbidity if not mortality and needs prompt recognition and therapy.

On the other hand, the most feared bleeding event is PPH (postpartum haemorrhage) in which there is an acute loss of more than $500 \mathrm{ml}$ blood at the time of delivery. It could have been predicted if the fibrinogen level is between 2 and $3 \mathrm{~g} / \mathrm{l}$ at the time of delivery. The risk was multiplied by almost 12 when the fibrinogen level was $<2 \mathrm{~g} / \mathrm{l}$, suggesting that fibrinogen had a positive predictive value of $100 \%$ for severe $\mathrm{PPH}$ at a threshold of $2 \mathrm{~g} / \mathrm{l}$ [5]. Immediate control of the bleeding source (surgically or by embolisation of bleeding vessel) followed by aggressive packed red cells transfusion and induction of the massive transfusion protocol (MTP) is the need of that hour. If the bleeding is not controlled, then additional components such as FFP / cryoprecipitate / platelets are used as a package to further control the coagulation process. It is better to initiate component therapy if the need is more than four packed red cells, especially if bleeding is ongoing, as depletion of blood factors can trigger coagulopathy. So preventing coagulopathy is better than letting it set in is the need of the hour.

There are several other micro-vascular coagulopathies such as eclampsia, eclampsia and HELLP (haemolysis elevated liver enzymes low platelets), HUS (haemolytic uraemia syndrome) and TTP (thrombotic thrombocytopenia purpura) due to microangiopathy in pregnancy that can significantly affect the pregnancy outcome.

A rising creatinine, thrombocytopenia with neurological features, fever and schistocytes on the smear suggest TTP, while similar features without a neurological involvement but with rising creatinine and anaemia suggest HUS. If the schistocytes are accompanied by rising liver enzymes and thrombocytopenia, then this is likely to be HELLP and if the new event is hypertension then this could be preeclampsia, especially with a rising LDH and proteinuria [6].

Mild preeclampsia was described in women with moderate hypertension and no more than a slight trace of albumin in the urine [7]. Severe preeclampsia (PE-SF) is now diagnosed when new-onset hypertension occurs $\geq 20$ weeks of pregnancy, in conjunction with at least one severe feature involving other organs such as the liver, kidney, eyes or lung by the ACOG's revised diagnostic criteria for preeclampsia 2013. The definitive treatment for preeclampsia and HELLP is delivery, after which maternal health usually improves steadily over 2-3 days [8].

TTP (thrombotic thrombocytopenic purpura) is a rare Thrombotic Microangiopathy (TMA) that is most often an acquired inhibitory auto-antibodies against ADAMTS13 (a disintegrin and metalloproteinase with a thrombospondin type 1 motif, member 13), an enzyme that normally cleaves Von Willebrand Factor (VWF) [9]. It can be diagnosed by a rapid onset thrombocytopenia, schistocytes on smear (fragmented red cells), anaemia with involvement of kidney (raised creatinine) and CNS (abnormal behaviour). In pregnant women that present with marked thrombocytopenia in the first half of pregnancy, TTP should be ruled out, because preeclampsia and HELLP are unlikely. Suspicion for TTP should also be high in those with personal or family history of TTP [10]. For women presenting with haemolysis and profound thrombocytopenia in the second half of pregnancy or postpartum, ADAMTS13 activity level should be sent to rule out TTP [6]. TTP is a rapid fulminant disorder that needs prompt recognition and specific therapy with plasmapheresis with or without steroids an additional agents. Particularly, platelet transfusion should be avoided as it can exacerbate the underlying disease in TTP and increase risk for neurological or cardiovascular events or death [11].

The historical association between TTP and HUS (haemolytic uraemic syndrome) has led to confusion and delay in the diagnosis of TTP. HUS is characterised by the 
triad of microangiopathic haemolytic anaemia, thrombocytopenia and acute kidney injury [12]. The treatment of HUS is dialysis/ plasma infusion / plasmapheresis / steroids just like in TTP and for the atypical variety is eculizumab [13]. All these can significantly impact the outcome of pregnancy adversely.

While the above-mentioned pathologies are at a microvascular level and hence not seen as an obvious feature clinically, a visually obvious isolated limb swelling with pain and colour change should warn us of a DVT (deep vein thrombosis) that can be confirmed on venous Doppler ultrasound of the involved limb. D Dimer is a diagnostic test for DVT, but in pregnancy it goes up as a natural prothrombotic protection against bleeding and hence not a good indicator of DVT as seen in the non-pregnant state. For this reason, a higher trimester-specific D-dimer cut-off could have a better sensitivity in pregnancy [14]. Pulmonary embolism (PE) could be easily missed due to commonality of symptoms with pregnancy; however, if there is persistent cough, diaphoresis, haemoptysis, syncope, fever, tachypnoea, hypoxia and hypotension she should be immediately attended to for PE. An undiagnosed and untreated DVT could affect the growth of the child and could cause a fatal pulmonary embolism. VTE (DVT with PE) accounted for $15 \%$ of maternal deaths reported in the USA between 2003 and 2011 [15]. No such specific data were published in Indian population in pregnancy. A V/Q scan (ventilation perfusion scan) is preferred over a CT pulmonary angiogram in pregnancy to diagnose pulmonary embolism because there is 150 times less breast radiation with a V/Q scan compared with a CTPA scan [16].

Once diagnosed, DVT / PE (with normal haemodynamics) needs urgent therapeutic anticoagulation with low-molecular-weight heparin $(1 \mathrm{mg} / \mathrm{kg}$ twice a day if the creatinine is normal) with a good safety profile as LMWH does not cross the placenta, and is not associated with any teratogenicity [17]. DOACs (direct oral anticoagulants) have not been studied during pregnancy or during breast feeding. In haemodynamic instability (hypotension) secondary to pulmonary embolism, thrombolytic therapy is the drug of choice and rtPA (recombinant tissue plasminogen activator) appears to be the safest fibrinolytic drug in pregnancy, with minimal placental transfer and no teratogenicity [16].

Postpartum, the VTE risk is highest in the first 3 to 6 weeks, but remains modestly elevated up to 12 weeks of postpartum [18] and caesarean section (CS) has an increased absolute VTE risk of 2.0 to 4.3 per 1,000 CSs [19]. Hence, extension of anticoagulation duration for 3-6 months postDVT/PE in such patients is beneficial to prevent DVT/PE at home when the patient is unsupervised! Decision of extension to prevent DVT at home is case based and on risk stratification. No such extension or prophylaxis is needed if there is no DVT or the patient is low risk and mobilised early.
Warfarin (with target INR 2-3 for desired duration) if used as extension or for prophylaxis post-delivery is safe during breastfeeding. It should be accompanied by low-molecularweight heparin till INR is $2-3$ on two consecutive tests.

A good ANC assessment, past/family history of bleeding or clotting events either in the pregnant state or otherwise along with the global coagulation profile /TEG will help diagnose many coagulopathies. Asking the pathologist or haematologist to view the peripheral smear for schistocytes as an early warning sign for microangiopathy helps provide appropriate early measures to salvage the pregnancy and the pregnant mother. Stopping anticoagulants in time prior to any surgical intervention or spinal/ epidural anaesthesia will prevent bleeding and reintroducing the anticoagulant post-op will prevent thrombosis for the high-risk patients.

Awareness and early detection of microangiopathies by the obstetrician will reduce morbidity and mortality. A timely referral to a physician or a haematologist will help sort out many of these adverse events and improve patient outcome. For patients who are in a less than tertiary care, a prompt transfer to a higher institution that has better facilities of specialists and blood bank could save lives.

\section{References}

1. Gogoi M. Pregnancy complications and birth outcome: do health care services make a difference? Int Res J Soc Sci. 2015; 4(3):27-35.

2. Bolte AC, Hermans FJ, Van Rheenen-Flach LE. [227-POS]: Thromboelastography (TEG®) and rotational thromboelastometry (ROTEM $\left.{ }^{\circledR}\right)$ in pregnancy: a systematic review. Pregnancy Hypertens Int J Women's Cardiovascular Health.. 2015;5(1):114-5.

3. Levi M. Disseminated intravascular coagulation (DIC) in pregnancy and the peri-partum period. Thromb Res. 2009;1(123):S63-S6464.

4. Erez O, Mastrolia SA, Thachil J. Disseminated intravascular coagulation in pregnancy: insights in pathophysiology, diagnosis and management. Am J Obstet Gynecol. 2015;213(4):452-63.

5. Cortet M, Deneux-Tharaux C, Dupont C, Colin C, Rudigoz RC, Bouvier-Colle MH, Huissoud C. Association between fibrinogen level and severity of postpartum haemorrhage: secondary analysis of a prospective trial. Br J Anaesth. 2012;108(6):984-9.

6. Gupta M, Feinberg BB, Burwick RM. Thrombotic microangiopathies of pregnancy: differential diagnosis. Pregnancy hypertension. 2018;1(12):29-34.

7. Irving FC. A study of five hundred consecutive cases of preeclampsia. Can Med Assoc J. 1939;40(2):137.

8. American College of Obstetricians and Gynecologists. Task Force on Hypertension in Pregnancy Hypertension in pregnancy. Report of the American College of Obstetricians and Gynecologists' task force on hypertension in pregnancy. Obstet Gynecol. 2013 Nov;122(5):1122-31.

9. Furlan M, Robles R, Solenthaler M, Wassmer M, Sandoz P, Lämmle B. Deficient activity of von Willebrand factor-cleaving protease in chronic relapsing thrombotic thrombocytopenic purpura. Blood. 1997;89(9):3097-103. 
10. Peyvandi F, Lavoretano S, Palla R, Feys HB, Vanhoorelbeke K, Battaglioli T, Valsecchi C, Canciani MT, Fabris F, Zver S, Réti M. ADAMTS13 and anti-ADAMTS13 antibodies as markers for recurrence of acquired thrombotic thrombocytopenic purpura during remission. Haematologica. 2008; 93(2):232-9.

11. Benhamou Y, Baudel JL, Wynckel A, Galicier L, Azoulay E, Provôt F, Pène F, Mira JP, Presne C, Poullin P, Halimi JM. Are platelet transfusions harmful in acquired thrombotic thrombocytopenic purpura at the acute phase? Experience of the French thrombotic microangiopathies reference center. Am J Hematol. 2015;90(6):E127-E129129.

12. N. James, A.C. George, Acquired TTP: Clinical Manifestations and Diagnosis, in: L.L. Leung(Ed.), UpToDate,UpToDate, Waltham, MA, 2017. Accessed on 22 Nov 2017

13. Bruel A, Kavanagh D, Noris M, Delmas Y, Wong EK, Bresin E, Provôt F, Brocklebank V, Mele C, Remuzzi G, Loirat C. Hemolytic uremic syndrome in pregnancy and postpartum. Clin J Am Soc Nephrol. 2017;12(8):1237-47.

14. Chan WS, Lee A, Spencer FA, Chunilal S, Crowther M, Wu W, Johnston M, Rodger M, Ginsberg JS. D-dimer testing in pregnant patients: towards determining the next 'level' in the diagnosis of deep vein thrombosis. J Thromb Haemost. 2010;8(5):1004-111.

15. Kuriya A, Piedimonte S, Spence AR, Czuzoj-Shulman N, Kezouh A, Abenhaim HA. Incidence and causes of maternal mortality in the USA. J Obstet Gynaecol Res. 2016;42(6):661-8.

16. Leonhardt G, Gaul C, Nietsch HH, Buerke M, Schleussner E. Thrombolytic therapy in pregnancy. J Thromb Thrombolysis. 2006;21(3):271-6.

17. Forestier F, Daffos F, Capella-Pavlovsky M. Low molecular weight heparin (PK 10169) does not cross the placenta during the second trimester of pregnancy study by direct fetal blood sampling under ultrasound. Thromb Res. 1984;34(6):557-60.

18. Kamel H, Navi BB, Sriram N, Hovsepian DA, Devereux RB, Elkind MS. Risk of a thrombotic event after the 6-week postpartum period. N Engl J Med. 2014;370(14):1307-15.
19. Blondon M, Casini A, Hoppe KK, Boehlen F, Righini M, Smith NL. Risks of venous thromboembolism after cesarean sections: a meta-analysis. Chest. 2016;150(3):572-96.

Publisher's Note Springer Nature remains neutral with regard to jurisdictional claims in published maps and institutional affiliations.

\section{About the Author}

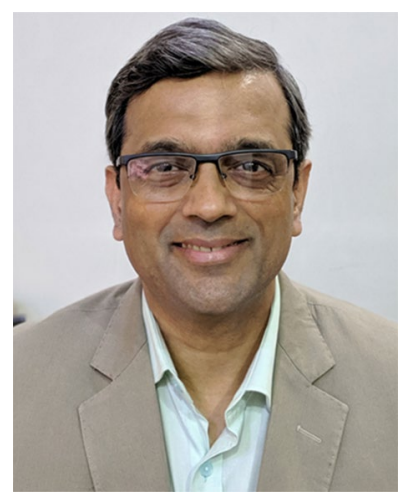

Dr. Abhay A. Bhave completed MD General Medicine from LTMMC, LTMGH, Sion hospital Mumbai. He was awarded Fellowship from Royal College of Pathologist of Australia, FRCPA, He is currently the Chief Editor of Lilavati hospital medical times, Section editor, hematology, the Indian Practitioner, Guest editor journal of Indian Medical Association. He is theICMR member for Myelodysplastic syndrome guidelines and is the recipient BR Ramasubramanian award by IMA for academic services. He is the panel member for Deep Venous thrombosis and Newer oral anticoagulants group, and Council member of Marrow donor registry of India 